\title{
ANGIOTOMOGRAFIA TRIDIMENSIONAL COMO EXAME PRÉ- OPERATÓRIO NO TRATAMENTO DOS ANEURISMAS CEREBRAIS
}

\author{
IVAN SOARES DE ARAUJO*
}

\begin{abstract}
RESUMO - O presente estudo se baseia em 36 casos do Departamento de Neurocirurgia da Universidade Livre de Berlim e procura mostrar as vantagens da angiotomografia tridimensional sobre a angiografia cerebral no tocante ao maior número de informações sobre os aneurismas, o que não ocorre com a angiografia cerebral que conta apenas com algumas incidências anatômicas limitadas: lateral, frontal e oblíquas. Com a angiotomografia tridimensional é possível a rotação de imagens reformatadas em $360^{\circ} \mathrm{em}$ todas as direções, conseguindo número considerável de projeções e mostrando o colo do aneurisma e sua relação com outros vasos e o fundo em relação com o colo. Também é demonstrável a relação do aneurisma com a parte óssea ao longo da base do crânio. A angiotomografia tridimensional tem-se mostrado tão sensível quanto a angiografia cerebral e recentemente tem sido usada no planejamento do acesso cirúrgico aos aneurismas cerebrais.
\end{abstract}

PALAVRAS-CHAVE: angiotomografia tridimensional, angiografia cerebral, aneurismas intracranianos.

\section{Three-dimensional computed tomographic angiography as preoperative examination for cerebral aneurysms treatment}

ABSTRACT - This study is based on 36 cases selected from the radiological clinic of the Free-University of Berlin and shows the advantages of spiral CT angiography over conventional cerebral angiography which provides only a few limited anatomical views: frontal, lateral and oblique. With CTA (computerized tomographic angiography) it is possible to obtain 360 degress reformatted images in all directions getting large number of projections and showing the neck of aneurysms and its relation to vessels and the fundus in relation to the neck. CTA also depicts aneurysms in relation to bone or portions of it along the base of the skull. The CTA achieves so good sensibility as the cerebral angiography does and is used as preoperative examination for the cerebral aneurysms treatment.

KEY WORDS: three-dimensional computed tomographic angiography, cerebral angiography, intracranial aneurysms.

Quando a angiotomografia helicoidal apareceu pela primeira vez em 1990, havia pouca evidência de que uma revolução na neuroimagem estava a caminho, possibilitando maior qualidade graças ao desempenho tecnológico que permitiu o aparecimento da angiotomografia tridimensional. A tomografia computadorizada e a ressonância magnética nuclear são exemplos de excelentes aplicações no desenvolvimento da neuroimagem. Por outro lado, a angiotomografia tridimensional tem menor custo, pronta disponibilidade e alta resolução espacial. A tecnologia de processamento de imagens está se tornando incessantemente importante na demonstração de grandes volumes de dados gerados pelos modernos "scanners" helicoidais. Aliás, a princípio, falava-se em angiotomografias helicoidais. Modernos "workstations" de gráficos permitem aos radiologistas explorarem

Desdobramento de projeto de pesquisa realizado em junho de 1996 no Departamento de Neurocirurgia da Universidade Livre de Berlim (Prof. Dr. Mario Brock): *Neurocirurgia do Hospital Universitário da Universidade Federal do Rio de Janeiro (UFRJ), Aluno de Pós-Graduação a nível de Doutorado em Neurocirurgia na Escola Paulista de Medicina da Universidade Federal de São Paulo. Aceite: 17-agosto-1998.

Dr. Ivan Soares de Araujo - Rua Assis Brasil 143/207 Bl. 3 - 22030-010 Rio de Janeiro RJ - Brasil. 
interativamente a alta qualidade das representações tridimensionais dos "data sets" da angiotomografia tridimensional. A aplicação deste tipo de tecnologia de computação para a orientação ao procedimento de invasão mínima constitui campo emergente que promete alterar radicalmente a maneira como a cirurgia vem sendo apresentada. A angiotomografia tridimensional também pode ser usada para a definição de aberrações anatômicas que são capazes de alterar o acesso operatório. Estudos recentes já começam a demonstrar a eficácia da angiotomografia tridimensional na avaliação diagnóstica de afecções cerebrovasculares ${ }^{1}$.

O planejamento operatório é um dos maiores beneficiados pelas técnicas de imagens tridimensionais. Enquanto o diagnóstico pode ser realizado a partir de cortes, as minúcias sobre o procedimento terapêutico exigem do cirurgião uma compreensão adequada da anatomia em três dimensões, como ilustramos com as imagens apresentadas neste estudo.

\section{CASUÍSTICA}

Foram estudados, do ponto de vista radiológico, 36 pacientes operados entre 1991 e 1995 . A pesquisa compreendeu estudo comparativo entre as imagens da arteriografia cerebral digital e das angiotomografia, tridimensionais, correlacionando-as com o diagnóstico operatório.

A técnica para a solução das imagens tridimensionais na angiotomografia tridimensional consiste na administração de constraste endovenoso iodado não-iônico (aproximadamente $60 \mathrm{ml}$ ). Uma sequência de cortes da área onde está o aneurisma é feita a intervalos de 40 segundos, enquanto a mesa, sobre a qual está o doente, move-se submetendo-se aos movimentos em espiral do "scanning" do tomógrafo. Através de "software" específico, obtém-se imagens tridimensionais dos cortes axiais coronais e sagitais reformatados. Através deste estudo é possível obter informações acerca do diâmetro externo do aneurisma, o coágulo no seu interior e sua relação com o parênquima cerebral, cisternas e ventrículos, que até agora não era possível conseguir na arteriografia cerebral. Outros detalhes incluem: forma do aneurisma, colo, projeção, bem como sua relação com vasos adjacentes e estrutura óssea ${ }^{2}$.

O estudo comparativo é ilustrado pelas imagens obtidas em casos analisados (Figs 1 a 5).

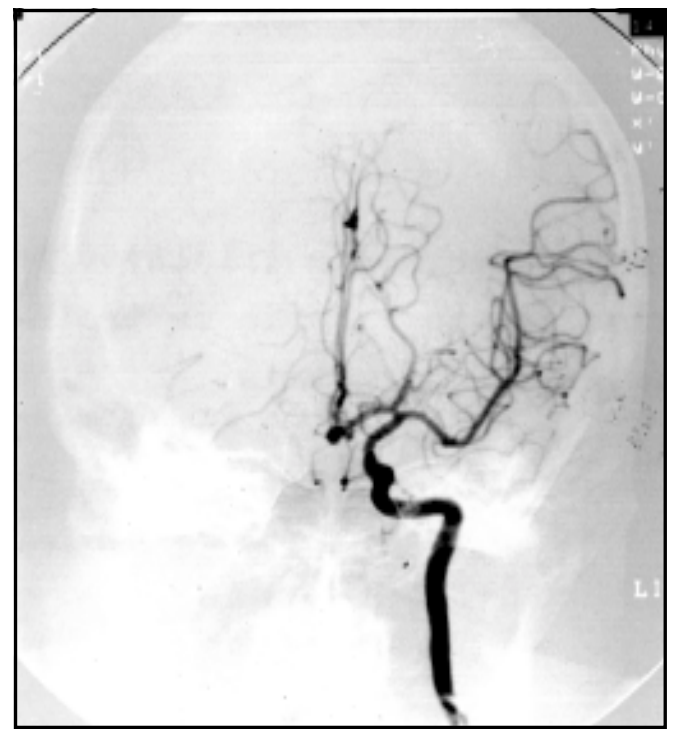

Fig 1. A angiografia cerebral ainda é o exame mais usado no pré-operatório dos aneurismas cerebrais mas só se usam três incidências anatômicas: frontal, lateral e oblíqua. Nesta paciente de 47 anos o aneurisma situa-se na artéria comunicante anterior. 


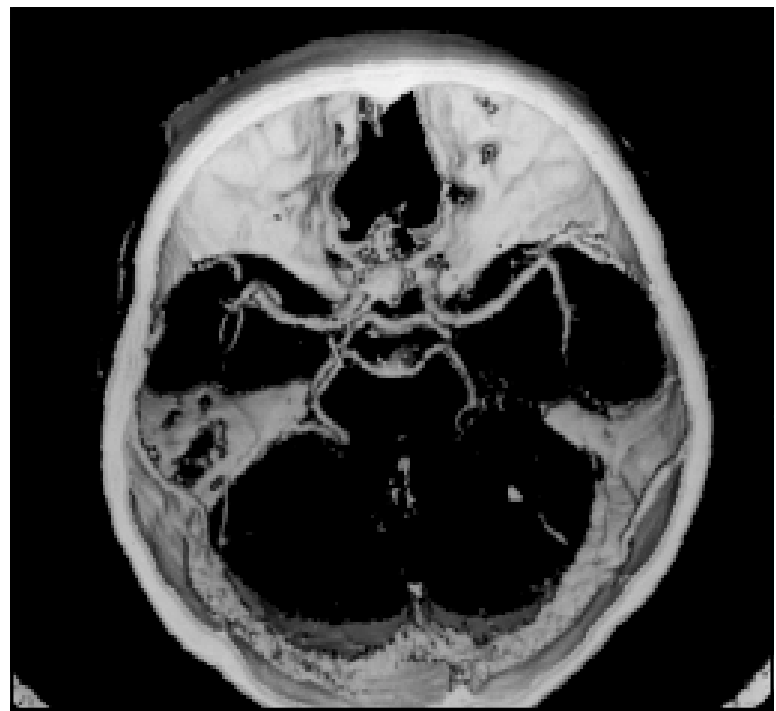

Fig 2. Na angiotomografia tridimensional, observa-se um aneurisma de situação idêntica à figura anterior.

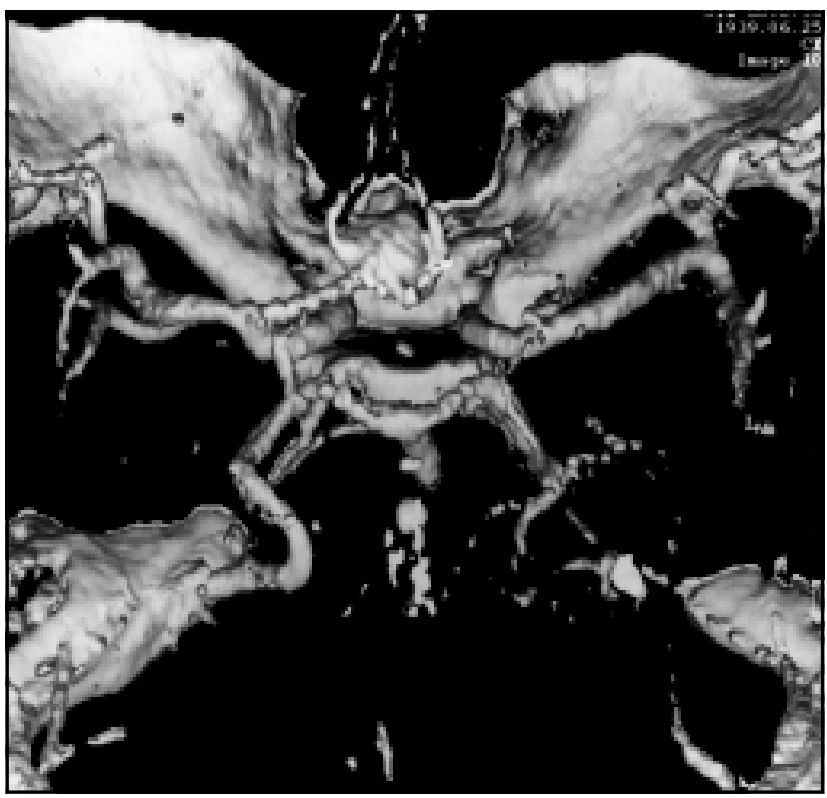

Fig 3. A angiotomografia helicoidal oferece mais informações ao neurocirurgião do que a angiografia que provê tão somente as incidências frontal, lateral e oblíqua. Aqui a angiotomografia tridimensional proporciona estudo mais abrangente, permitindo ver as relações do aneurisma com a partes ósseas e com outros vasos e até com o parênquima, aqui suprimido. 


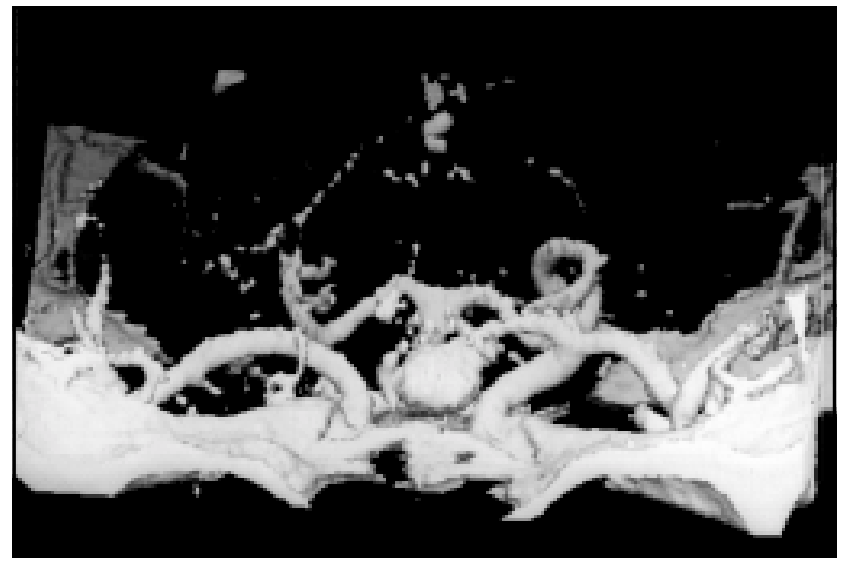

Fig 4. A angiotomografia tridimensional também desenha o aneurisma em relação ao osso ou parte deste ao longo da base do crânio. Isto permite ao cirurgião ver o que é possível remover para alcançar o aneurisma.

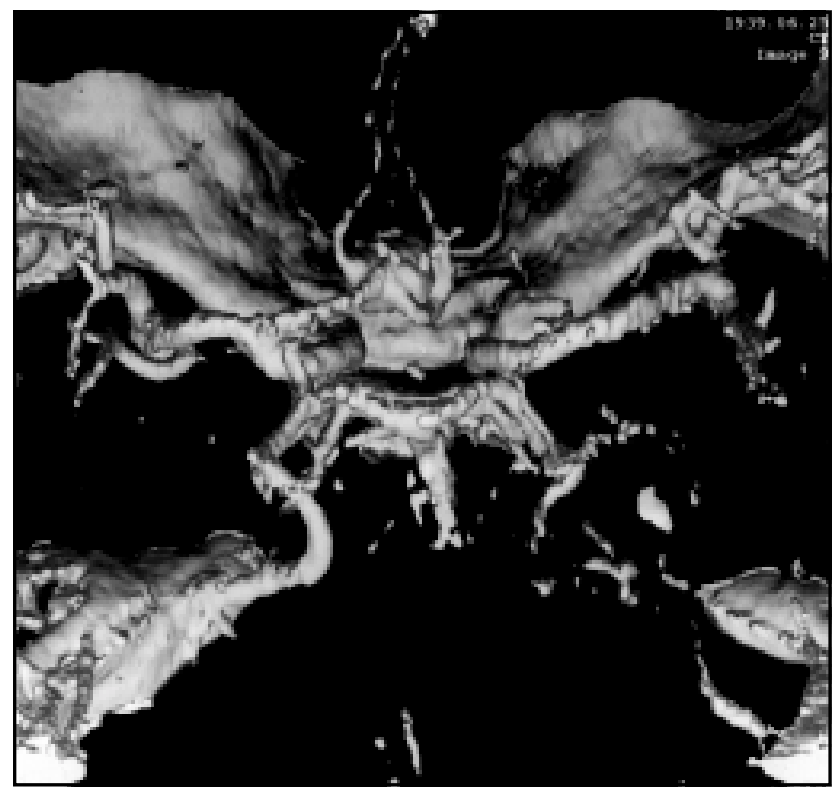

Fig 5. Aparentemente igual à Figura 3, aqui se observa a relação entre o colo e o fundo do aneurisma que é tipicamente da "pars rostralis" do polígono de Willis. Paciente do sexo feminino de 58 anos.

\section{DISCUSSÃO}

Um dos grandes trunfos da angiotomografia tridimensional é, sem sombra de dúvida, a capacidade de gerar um número considerável de projeções, permitindo demonstrar, numa anatomia tridimensional, o colo, o fundo e as relações anatômicas do aneurisma, o que é impossível na angiografia cerebral ${ }^{2-5}$. A ressonância magnética ainda não é páreo para esta modalidade de exame ${ }^{1}$. Os recursos técnicos gerados na formatação das imagens tridimensionais na angiotomografia ainda não foram empregados para 
angiorressonância. Para a quase totalidade dos neurocirurgiões, a angiotomografia é exame complementar da angiografia cerebral digital ${ }^{1}$. Há centros neurocirúrgicos, no entanto, que prescindem da angiografia cerebral para o planejamento de cirurgias cerebrovasculares ${ }^{2}$.

As vantagens da angiotomografia tridimensional, também chamada helicoidal, têm sido demonstradas ao longo dos poucos anos de sua existência. Neste estudo houve a preocupação de evidenciar aquilo que foi fruto da observação e análise do material: o confronto das imagens neurorradiológicas com a anatomia topográfica é de importância fundamental à compreensão das imagens tridimensionais ${ }^{5}$ e isto é observável compulsando obras de vulto sobre o assunto ${ }^{4}$ e publicações atuais ${ }^{6}$. Na época que antecedeu a tomografia computadorizada de crânio, a supremacia da angiografia cerebral era incontestável ${ }^{7,8}$. Mas, na atualidade há um pressentimento dos neurocirurgiões e neurorradiologistas de que a velha arteriografia de Egas Moniz e Almeida Lima está prestes a ser substituída por métodos incruentos como a angiorressonância magnética e angiotomografia digital tridimensional.

Não é próprio do neurocirurgião, mesmo nos grandes centros, prescindir da arteriografia cerebral para o planejamento da abordagem operatória do aneurisma intracraniano, mesmo com o aparecimento da angioressonância magnética nuclear e a contemporânea angiotomografia tridimensional. Os aprimoramentos tecnológicos e o conhecimento cada vez maior sobre a manipulação da informática têm revertido esta postura ortodoxa na propedêutica neurocirúrgica. Por outro lado, a falta de conhecimento adequado da anatomia topográfica tridimensional tem constituído outro obstáculo à aceitação desse novo recurso de neuroimagem, como exame pré-operatório. Escreveu Aloísio de Castro, em sua "Semiótica Nervosa": "Mas novidade é muita vez engano, tanto costuma o tempo vestir idéias velhas de novas aparências". Longe de assumir uma filosofia misoneísta, o grande mestre da Faculdade de Medicina da Praia Vermelha chamou a atenção, no seu tempo, para certas divulgações em foros científicos, à guisa de novidade, de certas associações nosológicas que nada apresentavam de excepcionais ou modernas. Em contrapartida, muitos procedimentos já foram apresentados em congressos e acham-se esquecidos; tal aconteceu com a leucotomia pré-frontal de Moniz-Lima e o implante de marca-passo no sistema nervoso central, dentre tantos outros exemplos.

Por fim, a angiotomografia tridimensional tem-se mostrado mais econômica e mais eficaz no estudo diagnóstico da anatomia vascular do que a angiografia cerebral ${ }^{1}$.

Em conclusão, a informação anatômica da angiotomografia é de valor inestimável e a arteriografia cerebral não passa de solução de duas dimensões para problema de três dimensões ${ }^{1}$. Ao planejar o acesso cirúrgico no tratamento de aneurisma de qualquer parte do polígono de Willis ou longe dele como em ramos distais da artéria pericalosa (aneurismas micóticos) ou na presença de hemorragia subaracnóidea espontânea, cujo estudo vascular nada revela de anormal, o cirurgião se sente muito mais beneficiado com as imagens geradas pela angiotomografia tridimensional do que com a angiografia cerebral ou a angiorressonância.

\section{REFERÊNCIAS}

1. Harbaugh RE, Schlusselberg, Jeffery R, et al. Three-dimensional computed tomographic angiography in the preoperative evaluation of cerebrovascular lesions. Neurosurgery 1995;36:320-327.

2. Laligan N, Seckhar, Kamal K, Kalia HY, Wrightdc, Ching H. Cranial base approaches to intracranial aneurysms in the subarachnoid space. Neurosurgery 1994;35:472-483.

3. Latchaw ER. MR and CT imaging of the head, neck and spine. 2.Ed. Saint Louis: Mosby Year Book, 1991:271-276.

4. Todury G. Anngewandte und Topographische Anatomie: ein Lehrbuch fur Studierend und Arzte. 4 Uberd. Stuttgart: Georg Thieme Verlag 1970:130-157.

5. Groot J. Correlative neuranatomy of computed tomography and magnetic resonance imaging. Philadelphia: Lea \& Febiger, 1984:87-91

6. Schirmer M. Neurochirurgie. München: Urban \& Schwarzenberg, 1994:242-259.

7. Krayenbuhl AH, Yasargil GM. Cerebral angiography. London: Butterworths, 1968:184-206.

8. Taveras MJ, Wood HE. Diagnostic neuroradiology, Vol III. Baltimore: Willians \& Wilkins 1976:911-952. 